Methods: Publications were identified by interrogating electronic databases; Medline \& MEDLINE In-Process, EMBASE and the Cochrane Library (accessed 6 Sept 2016). Eligibility criteria included adult patients with a diagnosis of acute/chronic gout or hyperuricemia, with no restriction on publication date, study design or geography.

Results: In total, 59 studies met the pre-defined inclusion criteria and were reviewed; of these, 17 reported the relationship between sUA levels and flares $(n=12)$ and/or tophus status $(n=11)$. Two studies were multinational (North America), and 15 were single country (US [ $n=10]$; Spain [n=2]; New Zealand $[n=1]$; Germany $[n=1]$; Japan $[n=1])$. The majority of studies had a follow-up period of $\leq 1$ year, with one reporting 10 years' follow-up. All 12 studies evaluating flares reported that achieving sUA levels $\leq 6 \mathrm{mg} / \mathrm{dL}$ was associated with a decreased risk of gout flares, compared with sUA levels $>6 \mathrm{mg} / \mathrm{dL}(\mathrm{p}<0.05$ in 8 studies). All 11 studies evaluating tophus status reported that achieving sUA levels $\leq 6 \mathrm{mg} / \mathrm{dL}$ was associated with improvements in tophus status, compared with sUA levels $>6 \mathrm{mg} / \mathrm{dL}(\mathrm{p}<0.05$ in 4 studies). The remaining 42 studies reported the impact of urate lowering therapy on sUA levels and gout flares or tophus status, but not the correlation between the parameters. The qualitative results in these studies indicated that increases in SUA levels were associated with an increased risk of gout flares and worsening of tophus status.

Conclusions: Maintenance of sUA levels $<6 \mathrm{mg} / \mathrm{dL}$ is associated with improvements in tophi resolution and flare reduction in adult patients with gout/hyperuricaemia. Whilst longer-term follow up studies (>5 years) are warranted, this review further supports that decreases in sUA levels are a marker for clinical improvements.

References:

[1] Keenan R, et al. Etiology and pathogenesis of hyperuricemia and gout. Elsevier Saunders. 2013;94:1533-53.

[2] Richette P, et al. 2016 updated EULAR evidence-based recommendations for the management of gout. Ann Rheum Dis. 2016:annrheumdis-2016-209707.

[3] Shoji A, et al. A retrospective study of the relationship between serum urate level and recurrent attacks of gouty arthritis: evidence for reduction of recurrent gouty arthritis with antihyperuricemic therapy. Arthritis Care Res. 2004;51(3):321-5.

Disclosure of Interest: S. Mitchell: None declared, L. Manounah: None declared, $\mathrm{H}$. Liedgens Employee of: Grünenthal GmbH, E. Johannes Employee of: Grünenthal $\mathrm{GmbH}$

DOI: 10.1136/annrheumdis-2017-eular.6023

\section{AB0897 FUNCTIONAL DISABILITY AND HEALTH-RELATED QUALITY OF LIFE IN CHINESE PATIENTS WITH GOUT: A CROSS-SECTIONAL STUDY}

T. Fu ${ }^{1}$, R. Yin ${ }^{1}$, L. Zhang ${ }^{1}$, Q. Zhang ${ }^{1}$, Y. Xia ${ }^{1}$, J. Ji ${ }^{1}$, Z. Da ${ }^{1}$, X. Zhu ${ }^{1}$, L. Li ${ }^{2}$,

Z. Gu ${ }^{1} .{ }^{1}$ Department of Rheumatology, Affiliated Hospital of Nantong University; ${ }^{2}$ School of Nursing of Nantong University, Nantong, China

Background: As the most common arthritis in adults, gout is a painful, inflammatory disease that may cause functional disability and decreased healthrelated quality of life (HRQoL). However, there are currently no known reported studies related to functional disability and $\mathrm{HRQ}$ oL of gout patients from China.

Objectives: This cross-sectional study aims to investigate the effect of demographic variables, disease parameters, and psychological status on functional disability and HRQoL of Chinese gout patients.

Methods: A self-report survey was administered to 226 gout patients and 232 healthy individuals using the Short Form 36 health survey (SF-36) for HRQoL and the Health Assessment Questionnaire-Disability Index (HAQ-DI) for functional disability. Gout patients were asked to complete the $10 \mathrm{~cm}$ Visual Analog Scale (VAS) for total pain, the Patient Health Questionnaire (PHQ-9) for depression, and the Generalized Anxiety Disorder (GAD-7) questionnaire for anxiety. Blood samples were taken to examine the level of uric acid (UA). Independent samples t-tests, Chi square tests, spearman and/or pearson correlation and multiple linear regression were used to analyze the data.

Results: Our results found that individuals with gout have poorer HRQoL compared to healthy controls and the mean disability score was 0.32 (SD 0.54), representing mild disability. SF-36 and almost all components of the SF-36 score were associated with place of residence, hypertension, DM, cardiovascular disease, disease duration, number of flares/last year, total pain, number of tophi, presence of tender joints, colchicines use, corticosteroids use, depression, and anxiety $(p<0.05)$. This variable was also significantly related to the HAQ-DI score $(p<0.05)$. Additionally, there were significant relationships among age, income/year, allopurinol use and HAQ-DI $(p<0.05)$. Stepwise multiple linear regression identified number of flares/last year, place of residence, depression and DM as predictors of functional disability. Disease status (total pain, number of flares/last year, presence of tender joints, cardiovascular disease, colchicine and corticosteroids use) and psychological disorders (depression and anxiety) were significantly accounted for poor HRQoL.

Conclusions: Chinese gout patients experienced mild disability and poor HRQoL. Disease status and psychological status were important risk factors linked to functional disability and HRQoL in Chinese gout population. These data suggest medical personnel should pay more attention to functional disability and HRQoL of gout patients and make suitable interventions to relieve their psychological disorders and finally to reduce their functional ability and improve their HRQoL.
Acknowledgements: This study was supported by Grants from the Chinese National Natural Science Foundation (no. 81671616 and 81471603).

Disclosure of Interest: None declared

DOI: 10.1136/annrheumdis-2017-eular.3078

\section{AB0898 SLEEP QUALITY IS ASSOCIATED WITH ALCOHOL USE AND FUNCTIONAL CAPACITY IN CHINESE PATIENTS WITH GOUT: A CROSS-SECTIONAL STUDY}

T. Fu ${ }^{1}$, R. Yin ${ }^{1}$, L. Zhang ${ }^{1}$, Q. Zhang ${ }^{1}$, Y. Xia ${ }^{1}$, J. Ji ${ }^{1}$, Z. Da ${ }^{1}$, X. Zhu ${ }^{1}$, L. Li $^{2}$, Z. Gu ${ }^{1} .{ }^{1}$ Department of Rheumatology, Affiliated Hospital of Nantong University; ${ }^{2}$ School of Nursing of Nantong University, Nantong, China

Background: Poor sleep quality is common in patients with chronic diseases and may lead to disease aggravation and decreased quality of life. The increasing prevalence of poor sleep in individuals with chronic medical conditions is associated with adverse demographic, clinical, and psychological characteristics. However, there are currently no known reported studies related to the sleep quality of gout patients.

Objectives: This study aims to evaluate the prevalence of poor sleep quality and investigate the contributors of poor sleep in Chinese gout patients.

Methods: A self-report survey was administered to 226 gout patients and 232 healthy individuals using the Pittsburgh Sleep Quality Index (PSQI) for sleep quality, the Patient Health Questionnaire (PHQ-9) for depression, and the Generalized Anxiety Disorder (GAD-7) questionnaire for anxiety. Gout patients completed the $10 \mathrm{~cm}$ Visual Analog Scale (VAS) for total pain, and the Health Assessment Questionnaire-Disability Index (HAQ-DI) for functional capacity. Blood samples were taken to examine the level of uric acid (UA). Independent samples t-tests, Chi square analyses, and logistic regression were used to analyze the data.

Results: Our results found that the prevalence of poor sleep (PSQI $\geq 5$ ) was $55.3 \%$ and the mean global score of PSQI was 6.69 (SD 3.48) in patients, which were significantly higher than the controls (17.7\% and 3.83 (SD 1.88), respectively). There were significant correlations among alcohol use, HAQ-DI, PHQ-9, GAD-7 and sleep quality in gout patients. Patients with yellow rice wine and wine use preferred to have better sleep quality. While, disease stage was associated with hypertension, total pain, number of tophi, presence of tender joints and swollen joints. Meanwhile, logistic regression models identified alcohol use and depression as predictors of poor sleep quality.

Conclusions: More than half of Chinese gout population suffered from poor sleep, which significantly higher than healthy individuals. These findings suggested medical personnel should pay more attention to the sleep quality of gout patients, especially those with depression. Additionally, it is beneficial for the patients with normal UA level to take moderate yellow rice wine and wine to improve their sleep quality.

Acknowledgements: This study was supported by Grants from the Chinese National Natural Science Foundation (no. 81671616 and 81471603).

Disclosure of Interest: None declared

DOI: 10.1136/annrheumdis-2017-eular.3081

\section{AB0899 DEPRESSION AND ANXIETY CORRELATE WITH DISEASE-RELATED CHARACTERISTICS AND QUALITY OF LIFE IN CHINESE PATIENTS WITH GOUT: A CROSS-SECTIONAL STUDY}

T. Fu ${ }^{1}$, H. Cao ${ }^{1}$, R. Yin ${ }^{1}$, L. Zhang ${ }^{1}$, Q. Zhang ${ }^{1}$, L. Li $^{2}$, X. Feng ${ }^{3}$, Z. Gu ${ }^{1}$ ${ }^{1}$ Department of Rheumatology, Affiliated Hospital of Nantong University; ${ }^{2}$ School of Nursing of Nantong University; ${ }^{3}$ Department of Stomatology, Affiliated Hospital of Nantong University, Nantong, China

Background: Depression and anxiety are common worldwide and may lead to disease aggravation and decreased health-related quality of life (HRQLL). The increasing prevalence of depression and anxiety in gout patients is associated with demographic and gout characteristics. However, there are currently no known reported studies related to the association between HRQoL and depression/anxiety.

Objectives: This cross-sectional study aims to evaluate the prevalence of depression and anxiety and investigate the potential risk factors for depression and anxiety in Chinese gout patients.

Methods: A self-report survey was administered to 193 gout patients and 208 healthy individuals from September 2015 to September 2016. Patients were asked to complete a set of standardized self-report questionnaires [Visual Analog Scale (VAS), Health Assessment Questionnaire-Disability Index (HAQ-DI), Patient Health Questionnaire (PHQ-9), Generalized Anxiety Disorder (GAD-7) questionnaire, Short Form 36 health survey (SF-36)]. Independent samples t-tests, $x^{2}$ analyses, and logistic regression were used to analyze the data.

Results: We found $15 \%$ of gout patients had depression, and $5.2 \%$ had anxiety, which were significantly higher than the healthy controls (1.4 and $1.0 \%$, respectively). There were significant correlations among education, pain, disease duration, stage of gout, disability, number of tophi, presence of tender joints, HRQoL, and psychological status. Meanwhile, logistic regression analysis identified number of tophi, HAQ-DI, and MH scale as predictors of depression in gout patients. Education, GH, and VT domains were significantly accounted for anxiety. 